\title{
U.S. OCEan ScIEnce NeEDS for Modeling AND DATA SyNTHESIS: STATUS OF A COMMUNITY ASSESSMENT
}

\author{
By Worth D. Nowlin, Jr.
}

B ECAUSE OF SEVERAL major factors, it is timely that the ocean science community examine its future needs for ocean models and data assimilation.

1. First computational capabilities and ocean general circulation models (OGCMs) continue to develop rapidly and apace. The U.S. community now has several OGCMs that might be considered to be community models in the sense that various scientists-not necessarily involved in their development-wish to utilize them. Other models still under development will reach this status. Initial efforts at ocean data assimilation have begun using these models. Biogeochemical processes are being added to ocean circulation models. and initial attempts at assimilation of biogeochemical data have begun.

2. Second, we have developed unprecedented capabilities to sample the ocean, although not continuously or with adequate global coverage of frequency. However. by-and-large we have not developed the ability to exploit the ocean data being collected by the large (e.g.. global change) research programs in sophisticated ways, such as via model data assimilation. These programs are at different stages of development/completion. One [Climate Variability and Predictability/Global Ocean-Atmosphere-Land System (CLIVAR/GOALS)] already is assimilating data in a quasi-operational research mode. Some [the World Ocean Circulation Experiment (WOCE) and the Joint Global Ocean Flux Study (JGOFS)] are nearing the synthesis phase with plans for model-data comparisons and model

Worth D. Nowlin. Jr., Department of Oceanography, Texas A\&M University. College Station, TX 77843-3146. USA. data assimilation. Others [Global Ocean Ecosystems Dynamics (GLOBEC)] are only beginning to plan their approaches to interpreting observations. But eventually, the need for synthesis using models must be faced by all large programs and all ocean science disciplines.

3. Third, satellite research missions are yielding some global data sets (e.g.. radiometry, scatterometry. and altimetry), in some cases beyond most aspirations. Other satellite missions will expand the types of measurements (ocean color, geoid, or surface property concentrations--perhaps even including surface salinity).

4. Finally, planning is underway to increase satellite monitoring missions and long-term in situ measurements as contributions to observing systems [Global Ocean Observing System (GOOS) and Global Climate Observing System (GCOS)] for a variety of practical applications and to underpin future research and development.

\section{Questions/Needs}

In this environment, the ocean science community should address the following questions (among others) regarding ocean modeling and data synthesis.

1. Are new structures or resources needed to provide for the maintenance and further development of ocean models and for their broad use by the community? (More specifically, what is needed to ensure model diversity and for further model development and testing-some ocean disciplines have few if any models satisfactory to their needs. What needs exist for the care of ocean models that have reached the level of community models? What is needed to allow access, use, and modification of such models by the community of users?)
2. What capabilities are needed to best employ models in the synthesis of ocean research data? Although this question is most pressing for the large research programs nearing data synthesis phase, it should be of interest to a broad segment of the community. We are now collecting many data sets large in scope (either in space-time or in diversity). However, it seems likely that our sampling will always be too limited in space, time. and parameters to completely describe complicated ocean phenomena and processes using observations alone. Models must be employed to integrate and interpret the data sets, consistent with reasonable constraints.

3. How do models contribute to the design and implementation of long-term observing systems? We wish to have these systems designed to best capitalize on long-term ocean observations for improved understanding, as well as for products useful to society. It seems certain that most of such products will be the result of combining observations with models. Moreover, examination and use of such products will provide a key means for improvement of the observing systems.

\section{The WOCE Experience}

Let me convey the experience of WOCE with data assimilation. WOCE planning began in the early 1980 s, before serious thought was given by the ocean science community to data assimilation as a major mode for synthesizing observations. This need has become clear during this decade.

Starting about four years ago, contacts were made with managers in federal funding agencies [Navy, the National Science Foundation (NSF), the National Oceanic and Atmospheric Administration (NOAA). the Department of Energy (DOE), and the 
National Aeronautics and Space Administration (NASA)] to obtain access to a major computing facility at which the capability for model synthesis of the WOCE data could be developed. This would require adding to an existing computational capability the access to data and gridded data sets and a team of experts in ocean modeling and observations. Not having received clear positive responses, WOCE organized a meeting of agency representatives at NSF in fall 1996.

At that meeting, agency representatives made quite clear that existing laboratories with computational facilities adequate for the task are rather fully committed to agency missions. It also was clearly expressed that the capabilities needed by WOCE would also be needed by other large ocean research programs, and perhaps by the general ocean science community of the future.

\section{An Opportunity}

Triggered by that meeting, the community was offered an opportunity by the NSF Ocean Sciences Division, directed by Mike Purdy. On August 30, 1996. Purdy sent me a message stating:

... we believe that an important component of the future global ocean sciences is the creation of the infrastructure and environment in which data assimilation, integration. modeling, and interpretation of large diverse data sets can take place. This is an issue that extends well beyond WOCE and we encourage you to lead a community-based effort to provide us with advice concerning the form that support for such capability should take. We consider it important to design a model that can sustain growth over a substantial period of time because it is inevitable that the beginnings will be modest, but the requirement will grow substantially over the next decade.

It is logical that WOCE should provide the leadership for this activity, but it is important that the effort be . . a service to the community as a whole. . . . Would it be useful, perhaps, to form a small high-level "executive" steering group . . . to serve as a leadership group and attempt from the start to establish the broad nonpartisan nature of this initiative?

In recognition of the broad-based nature of this planning activity we . . . would supplement it at the Division level. . . .

After that message, an ad hoc steering committee for ocean modeling advances consisting of Andrew Bennett (community at large), Russ Davis (Ocean CLIVAR), Hugh Ducklow (JGOFS), Worth Nowlin (WOCE), Thomas Powell (GLOBEC), and Doug Wallace (DOE carbon dioxide program) was established in September 1996. A series of community discussions to assess requirements for future computational work by the ocean science community was considered.

The first such meeting, held in Dallas, TX on 20-21 February 1997, dealt with ocean data assimilation activities and requirements. The second, held in Boulder, CO on 7-8 April 1997, focused on community needs for ocean general circulation modeling. Results of those meetings were presented and discussed at a meeting in Irvine. CA on 3-4 June 1997 of the Committee on Major U.S. Oceanographic Research Programs (reporting to the Ocean Studies Board of the National Research Council). Results of these meetings are summarized in the next three sections.

\section{Results of Ocean Data Assimilation Workshop}

The intent of the February meeting was to begin the process of exploring community requirements for data assimilation and to discuss whether a community ocean data assimilation center is required.

Attending the meeting were representatives of CLIVAR, the Coastal Ocean Program, the DOE Carbon Dioxide program, GLOBEC, JGOFS, Ocean Drilling Program, Ridge Inter-Disciplinary Global Experiments, WOCE, and NASA and NOAA laboratories, as well as several unaffiliated individual scientists. The meeting was open, and an announcement was published. Background papers were solicited and distributed prior to the meeting.

Time was allotted at the meeting for all attendees to make statements regarding their interests, requirements (or those of their laboratory or program). and aspirations for the meeting. There followed a series of four presentations on general elements of ocean data assimilation (Andrew Bennett), shelf models (Keith Thompson), quasi-operational El NiñoSouthern Oscillation (ENSO) prediction modeling (Dave Behringer), and basin/ global ocean modeling (Jochem Marotzke). These talks, as well as brief plans and requirements of specific programs and laboratories represented, are summarized in the report of the meeting (U.S. WOCE Office, 1997a).

The discussions focused on the themes:

- Common requirements,

- Special requirements for biogeochemical studies,

- Components (infrastructure) needed for ocean data assimilation,

- Sociological factors to be considered,

- Technical aspects of data assimilation.

Workshop participants represented many disciplines and interest in widely varied scales. Nevertheless, it was clear and considered important to stress there is a broad dynamic range of modeling activities common across the ocean sciences community. The common requirements identified by the meeting as being important to future data assimilation efforts were principally related to data, models, products and users. or sociological factors.

\section{Regarding Data}

Continuing data. Without a data stream there can be no ongoing data assimilation. Thus there is the need for data gathering operations to continue.

Timely data access. Timely access to data by modelers is a prerequisite for a successful data assimilation effort. Data must be considered as being common to all, rather than being held by individual researchers.

Estimates of data error and scales. Data assimilation requires more than just data sets. These need to be sampled and organized in such a way that data assimilators can obtain correlation forms and scales for each variable observed as well as noise estimates.

Retrospective analyses. Needed are continuing analyses of data sets to provide historical perspective with error bars.

\section{Regarding Models}

Continuing model development. Continuing model development is needed. including the development of new codes, priors for each model, and specific mathematical investigations related to these. New methods for comparisons between data and model output must be investigated.

Community models. Several models must be supported as community models. No attempt was made to define which or how many are required. 
Assess computational requirements. There is a need to assess the computational requirements for present and future operations in this area. This was beyond the scope of the meeting.

\section{Regarding Users and Products}

Users. It was felt that strong focus should be placed on users of model products. Of course. initial users likely will be researchers. However, it is important to plan from the beginnings of organized ocean data assimilation for the accommodation of a broader range of users.

Products. With regard to products. consider the impacts of the Comprehensive Ocean Atmosphere Data Set. Levitus and other climatologies, and similar products on research modeling. Clearly such analyzed fields will be primary products of data assimilation, but there is the early need to ask what products can be produced in the course of research that might be of value to nonresearch elements of society as well as to researchers.

\section{Regarding Sociological Aspects}

Education. Teach people, by example, what to expect of data assimilation. Objective interpolation provides a framework; but, most people don't completely understand the limitations/drawbacks of analysis by objective interpolation. It will be a large next step to interpret the evolution of sets of maps (representing states of the ocean) to better understand why such changes occur.

Improved leadership. There is a need for excellent leadership for new efforts such as an ocean data assimilation center to ensure that the objectives are reasonable. Community leadership must be joint between agencies and academia.

Connectivity: The community must establish and maintain active connections between modeling groups within diverse programs and disciplines, and also between modeling and data centers.

Design of observations. Ocean data assimilation has a natural role in planning/design for new field programs and long-term observing systems. Most products from such systems will be model generated.

After considering common needs, the meeting discussed desirable characteristics of a national ocean synthesis activity. It was agreed that this activity must:

- Emphasize need for critical levels of effort;

- Adopt an interdisciplinary outlook;
- Focus on products (e.g., provide useful results).

Elements of the activity must include:

- Ready access to multiple community data sets, products, and climatologies. It is not necessary for the center to be situated at a major data center, but there must be good networking links between them:

- Capability to run several community models at different resolutions (e.g.. global, coastal, and biogeochemical models):

- Ready access to the latest technology for data assimilation;

- Critical scientific and support mass;

- Centralized computing necessary for long-term computations;

- Strong outreach and visitor programs.

It was generally agreed that the ocean science community would greatly benefit from the capabilities available through an ocean data assimilation center. The probable first step should be production of products based on one or more models of the global ocean at a particular resolution. Successful physical models already are evolving to incorporate biogeochemical and biological processes. Such global and basin models could be seen as a framework for supporting nested coastal models. The evolution of states of the ocean could be simulated. Forecasts would remain the longer-term goal. The recommendation was that the community work to establish such a center, but the nature of the center (activity) was not specified.

It remains a task for the U.S. ocean science community to make specific the requirements in this area of activity.

\section{Results of Ocean General Circulation Modeling Workshop}

On 7-8 April. 1997, a workshop organized by Dale Haidvogel, James McWilliams, Rainer Bleck, and Ken Denman was convened at the National Center for Atmospheric Research (NCAR) in Boulder, CO. The focus was community needs in support of ocean general circulation modeling. Invitations were sent to representatives of the CLIVAR, GLOBEC. JGOFS, and WOCE programs, to DOE. NASA, and NOAA laboratories (scientists and laboratory mangers), and to individual model practitioners.

McWilliams began the meeting with a set of premises to guide discussion. He welcomed discussion of organization, resource needs. and financing of OGCMs. including the topics of model development, model testing, computations, solution analyses, and data comparisons. The focus was on models for calculating global equilibrium states and natural variability on time scales from weeks and longer; this encompasses model uses for climate, ocean diagnoses and data assimilation, prediction, and biogeochemical cycles.

Specific purposes addressed by the workshop participants were:

- To assess the current and future uses and resource requirements of OGCMs.

- To define procedures whereby institutionally supported models can be used and contributed to by a user community of academic scientists, including the possible adoption of new codes and algorithms.

- To promote a higher national priority and level of funding of OGCM development and testing (across the federal agencies supporting such modeling: NSF, NOAA, NASA, DOE, and ONR).

- To achieve better institutional support for a few community models, including the infrastructure requirements for staff, computers, data sets, and code maintenance.

- To define a process for selecting community models, both now and in the future.

- To identify requirements and developments necessary for effective use of community OGCMs in biogeochemical applications.

Following talks by Jochem Marotzke. Ken Denman, and Lynne Talley on future requirements for modeling as part of large global programs. representatives of several modeling groups described their model evolution and intended development. This is summarized as Section III of the meeting report (U.S. WOCE Office, 1997b). Summarized in Section V is a presentation by Albert Semtner on the present status and future requirements for computational resources devoted to OGCM studies.

The principal discussions focused on the need for continued support of OGCM studies. The consensus was that the national ocean modeling community must integrate and coordinate its presently piecemeal efforts at model development, validation, and comparison. if the large- 
scale scientific and programmatic goals of the previous section are to be met within realistic levels of resources and effort. Mechanisms need to be developed to encourage ocean modelers to pool their efforts, and to provide a stable and sufficient funding base for such activities. To this end. the meeting participants endorsed the concept of a "Consortium for Community Ocean Models" (CCOM).

As an example of a cooperative modeling activity, the Community Modeling Effort in WOCE was cited. Participants believed that the consortium could assume important, new roles as an endorser and active spokesman of community modeling activities with respect to the funding agencies. The structure would go beyond disconnected individual proposals and enable individual investigators to be funded for work on community-defined problems that are required to reach major technological goals.

The CCOM should (1) address largescale scientific problems. e.g.. North Atlantic Oscillation. ENSO/Pacific-North America oscillation. or global change forcing, and (2) adopt a comprehensive (multidisciplinary, whole-system) modeling approach. More specifically, the Consortium will exist to:

- Coordinate efforts to develop and refine numerical models for use in world ocean circulation, climate, and biogeochemistry simulations;

- Coordinate experiments with such models so as to optimally explore the pertinent parameter spaces and provide benchmark solutions;

- Provide infrastructure (personnel for technical support. computing resources. network access, data storage capabilities, meeting and travel funds) to allow mature models to reach the status of true community models;

- Develop versions of these models that serve the specific needs of the biogeochemistry and (coupled) climate modeling communities:

- Develop observational analysis products for forcing, initialization, assimilation, and evaluation of model solutions;

- Promote cooperation between ocean modelers and computer scientists and engineers to ensure that models make efficient use of modern computer architectures;

- Facilitate community access to model output to encourage scrutiny of model results in the light of observational evidence:

- Create test beds for innovative algorithms that promise to enhance the computational efficiency or physical correctness of existing models.

CCOM should be viewed as a parallel to the large field programs, with which it will share the responsibility for accomplishing their scientific goals. As envisioned, CCOM will consist of a Scientific Steering Committee (SSC) and several Science Teams. These teams will consist of researchers from universities and the large mission laboratories active in OGCMs: each will have a particular class of OGCMs as its focus. The meeting identified the fixed grid ( $z$, sigma) or "level model" family, and the isopycnic or "layer model" family of ocean models as initial model classes for Science Teams because established development and user communities exist for both these model families. CCOM should use the Science Teams to encourage a diversity of scientific approaches and allow for continuing evolution, or perhaps an occasional revolutionary succession, in the standard ocean models.

The summary of this workshop (U.S. WOCE Office, 1997b) suggested that this recommendation be widely circulated and considered by the community, and, that if sufficient support is found, the NSF Ocean Sciences Division appoint a provisional steering committee to develop a CCOM implementation plan. I would suggest that an alternative approach, more likely to happen, is for model practitioners to take initiatives needed to solve existing organizational problems.

\section{Discussion of Committee on Major U.S. Oceanographic Research Programs}

The status and requirements for ocean data assimilation modeling were a principal focus of the third meeting of the National Research Council's Committee on Major U.S. Oceanographic Research Programs on 3-4 June 1997 in Irvine. CA. The discussions began with five presentations. Worth Nowlin reviewed reasons for assessment of community requirements regarding ocean modeling and data assimilation and summarized the two community workshops on these topics. Tony Busalacchi described NASA/community efforts in ocean/atmosphere data assimilation, highlighting the Data As- similation Office; results, problems, and plans were reviewed. Eileen Hofmann reviewed ocean ecosystem data assimilation efforts to date. comparing alternative approaches to assimilating biological with physical data, and reviewing major difficulties and consequent requirements. Detlef Stammer presented and evaluated results of a preliminary attempt at global assimilation of one year TOPEX/POSEIDON data set into an OGCM. Russ Davis reviewed requirements of Ocean CLIVAR for long-term observations, modeling, and data assimilation.

Potential structures for an ocean data assimilation "center" (or ocean data synthesis activity) were discussed. One apparently acceptable structure suggested is similar to that of the European Center for Medium-Range Forecasts and that originally intended for the International Research Institute for Climate Prediction. A single hub has 1) massive computing capability and 2) capabilities for data ingest and quality control. It is engaged in ocean data assimilation for such purposes as improving technical capability, data quality control, and reanalysis. The hub has financial support to assist cooperating scientists not colocated (outreach program) as well as for a strong visitor program.

The hub is connected to a group of centers (nodes) focused on more specific aspects of data assimilation, e.g., ecosystem development and use of ecosystem models, $z$ - or sigma-coordinate models, mixed-layer models, coupled models. observation system simulation experiments for program design, or nested models. Computing power and data would be available to the nodes via the hub.

Modes of funding and organization of such a structure were discussed. Clearly it is important that the centralized portion (hub) remain responsive to general community needs. A directorate representing the institutions involved in the community (perhaps similar to the University Corporation for Atmospheric Research) may be required to provide oversight and ensure commitment of resources to broad community needs.

The major ocean research programs have created awareness of the needs for large data collection activities and for data assimilation capabilities. However. even though the scientists most prepared at this time to use an ocean data assimilation center are those in major ocean research programs concerned with general 
ocean circulation and relations to climate variability, it seems inevitable that the major use of such capability will soon be by coastal/regional/biogeochemical researchers. Many if not most users ultimately will not be associated with major ocean research programs.

The Global Ocean Data Assimilation Experiment (GODAE) proposed by the Ocean Observations Panel for Climate (of GOOS, GCOS, and World Climate Research Programme) was briefly discussed. Other nations are considering massive ocean data assimilation capabilities, particularly focused on satellite retrievals (e.g.. France). The question was raised "If most ocean data assimilation is being done by other countries, will observation systems follow?"

The next section describes the proposed GODAE. It might be considered as a test of both concepts of global observing systems and of centers for quasi-operational ocean data assimilation. For more information see http://www.bom.gov.au/ $\mathrm{bmrc} / \mathrm{mrlr} / \mathrm{nrs} / \mathrm{oopc} / \mathrm{godac} / \mathrm{homepage}$ html.

\section{A Global Ocean Data Assimilation Experiment}

The Ocean Observations Panel for Climate has the view that attracting the resources necessary for the long-term sustainability of a global ocean observing system for climate depends on a clear demonstration of the feasibility and value of such a system. As a demonstration, that Panel has proposed a Global Ocean Data Assimilation Experiment.

Many elements of a global ocean observing network are either in place now or the technology exists with which to develop and implement them. Progress in modeling, data assimilation, and computational power means global data assimilation at eddy-resolving scales should be feasible within a 3- to 5-y time frame. It is likely that by the start of the next decade several research groups will have performed initial global data assimilation experiments. GODAE is a proposal to bring remote and in situ observations, modeling, and data assimilation all together in a demonstration of the practicality of regular, routine, real-time global ocean data assimilation and prediction.

The fundamental objective of GODAE is to provide a practical demonstration of real-time global ocean data assimilation in order to provide a regular, complete depiction of the ocean circulation at time and space scales from several days and tens of kilometers to those of climate.
The resulting descriptions would be consistent with a suite of space and direct measurements, as well as with appropriate dynamical and physical constraints. The prime subobjectives are to 1) extend predictability for regional and coastal systems, 2) forecast upper ocean currents and temperatures for several days to several weeks. 3) provide initial conditions for climate prediction, 4) establish a continuing analysis framework and foundation for science (e.g., ecosystem studies). and 5) provide motivation and pathway for the establishment of a permanent global ocean observing system.

Foundations on which the experiment will be designed and carried out are the observing networks, models and assimilation, and scientific knowledge-these are discussed briefly in the following paragraphs.

Added to the operational or near-operational in situ observing networks, it is now becoming clear that the space programs will deliver a global observing system providing adequate ocean surface measurements (in terms of accuracy and sampling) for the period from 2000 to 2010. Although such measurements are very powerful, they are limited because they are not adequately integrated with other measurement systems nor with modern data assimilation systems and cannot by themselves provide three-dimensional sampling of the ocean. Through various research programs, a wide range of direct observations are available for calibration and for providing information on the vertical structure and circulation of the oceans. Experimental observational studies that will benefit the present project include those being conducted in the North Atlantic at present as part of various programs.

The feasibility of modeling the global ocean circulation at eddy-resolving scales has been demonstrated. There have also been several studies of global data assimilation, although usually not at the scales of eddies, and certainly not for real-time temporal resolutions of the order of several days. In other words, the potential for real-time global model data assimilation exists but, due to resource and knowledge constraints, there is as yet no demonstration of the practicality of such an undertaking.

It is obvious, although sometimes overlooked, that adequate knowledge of the variability and dynamical and physical processes taking place in the real ocean are vital for the success of an experiment such as that proposed. It is impossible to draw full value from observations, or to exploit synergy between different components of the observing network, without this knowledge and understanding. Assimilation should be seen not only as a method for transferring knowledge from the observables to the state variables of the model, but also as a tool for developing and enhancing our knowledge of the ocean circulation and variability (both of the explicitly resolved fields and of the unresolved noise/ residuals).

GODAE will work in cooperation with other regional and related global observational and research programs, including ENSO forecast systems. WOCE data synthesis, and the emerging research initiatives of CLIVAR, among others. At this point the timing and duration of the experiment remain relatively flexible, depending in part on the plans of space agencies and in part on our ability to realize an appropriate direct observation network. There are five critical phases: 1) definition of the experiment: 2) feasibility studies and scoping; 3) testing model and assimilation subcomponents, global prototypes. data assembly methods, real-time communications; 4) assemblage of subcomponents: and 5) realization of operational global ocean data assimilation systems (at least several "centers" of activity are envisioned).

Support for GODAE has been obtained from the Committee on Earth $\mathrm{Ob}$ servation Satellites (CEOS) Strategic Implementation Team. The space agencies, through CEOS wish to proceed rapidly on the development of this proposal; the Centre National d'Etudes Spatiales has been designated the lead CEOS agency for this proposal. The schedule is critical. For the space-based component, instrument schedules are already being locked in place for the first decade of the next century. A good tactic is probably to take these as given. which would point to the years 2002 to 2007 as a good time for this experiment.

Planning is proceeding, and the United States must take a role in this experiment or fall behind in this fundamental new approach to dealing with ocean data for research and for more utilitarian products. Certainly, this activity must be kept in mind when planning for the longer term data assimilation needs of the U.S. oceanographic community. 


\section{Planning the Next Steps}

On 5 September 1997. a meeting was held at the NSF in Arlington, VA. The meeting objectives were 1) to review and discuss recent actions to assess community needs for ocean modeling and data assimilation and 2) to recommend the next steps to firm up community requirements. Attendees included steering committee chairs of most of the U.S. major ocean programs, modelers, and representatives from the NSF Ocean Sciences Division and NASA.

The meeting participants agreed on several general points. There exists a continuum of needs for ocean data assimilation, from research to operational. Model and data assimilation capabilities are at different stages of development within different programs, disciplines, and subdisciplines. The role of physical oceanographic modeling is critical to the development of capabilities in other disciplines. The community needs structures and partnerships for ocean data-model interactions, as stated in the reports of the two previous workshops.

The need was endorsed for an ocean sciences model data assimilation system with the following broad objectives:

- Encourage diagnosis of observed ocean changes.

- Encourage hypothesis/scenario testing.

- Encourage intra- and interdisciplinary exchanges of models and data assimilation techniques,

- Promote use of models and synthesis for education and training,

- Improve efficiency of presently diffuse activities.

The system envisioned to meet these needs was very similar to that suggested at the June 1997 meeting of the Committee on Major U.S. Ocean Programs, de- scribed briefly in an earlier section. It would consist of a hub and nodes. The hub would have large computational capability, input/output providing access to (near) real-time data and to historical data sets, a cadre of experts. and a visiting scientist/outreach program. Nodes would consist of individual scientists or scientific teams at home institutions engaged in specific aspects of modelingdata interactions. Activities at nodes should develop new models and apply them using hub capabilities as needed. The objectives of these node activities will change in time and will be selected by the peer review process. Hub capabilities should be ongoing and so must be block funded. Oversight is needed to ensure that the hub be responsive to needs of the broad community, and that it does not grow to include a large scientific complement in competition with scientists working at nodes.

This system was discussed at some length. Capabilities needed at the central hub were identified based on support needed for tasks likely to be carried out by the community via the nodes. It was thought that a carefully designed system could meet the requirements enunciated in both prior workshop reports as well as other needs.

It was agreed that a broad-based community workshop to focus the needs for and design an ocean sciences model data assimilation system will be held 4-5 May 1998. Funding will be provided by the NSF Ocean Sciences Division, with logistics support by the U.S. WOCE Office. It will be organized by Thomas Powell and Worth Nowlin with advice from attendees at the 5 September meeting and others in the community. Representation will include the various modeldata activities underway throughout the community as well as the major ocean programs.

\section{Concluding Remarks}

I believe that the ocean science community is moving in the direction of building a strong case for a national ocean data assimilation system. Such a synthesis activity would fill a present gap in the research capabilities of the ocean science community. Needed now is agreement on specific requirements. As these requirements are being specified, a close watch should be maintained on the proposed GODAE- that activity could provide needed experience, or even new U.S. capabilities and motivation.

This same system might support development and community use of ocean models. This should not be limited to general circulation models, but perhaps begin with them, because of their more advanced state of development. I believe that practitioners combining data and models must take the initiative in devising and initiating the needed structures.

The NSF is concerned about general needs of the community in ocean modeling and data assimilation and has offered to provide the resources needed to define the requirements. The next steps are up to us in the ocean science community. There will be an ocean model data assimilation system workshop during the spring of 1998 where discussions will continue.

\section{References}

U.S. WOCE Office, 1997a: Report on Workshop to Discuss Needs for an Ocean Data Assimilation Center. Dallas. February 1997. College Station, Texas, 18 pp.

1997b: Report on Workshop to Discuss Community Needs for Ocean Global Circulation Modeling. Boulder, April 1997, College Station. Texas. $12 \mathrm{pp}$. 